VoL. 76 (2007) [195-204]

\title{
PERTURBATION THEORY OF MULTIVALUED ATKINSON OPERATORS IN NORMED SPACES
}

\author{
Teresa Álvarez and Diane Wilcox
}

\begin{abstract}
We prove several stability results for Atkinson linear relations under additive perturbation by small norm, strictly singular and strictly cosingular multivalued linear operators satisfying some additional conditions.
\end{abstract}

\section{INTRODUCTION}

The perturbation problem in Fredholm theory refers to the stability of the semiFredholm operators under additive perturbations. This problem has been around for many years. Its origen can be traced back to some classical results due to Kato and Vladimirskii. Let $T$ and $K$ be continuous operators acting from a Banach space $X$ into a Banach space $Y$. In $[9,2]$, Kato proved that $T+K$ is upper semiFredholm whenever $T$ is upper semiFredholm and $K$ is strictly singular. Similarly, in $[14,1]$, Vladimirskii proved that $T+K$ is lower semiFredholm if $T$ is lower semiFredholm and $K$ is strictly cosingular. Subsequently, Müller- Hörrig [10] and González [6] proved the analogous results for Atkinson operators in Banach spaces, that is, semiFredholm operators with complemented null space and range.

Atkinson linear relations in normed spaces are introduced in [3], where the authors obtain characterisations theorems of such multivalued linear operators in terms of the existence of left and right generalised inverses. In this paper, these characterisation theorems will be used in conjunction with perturbation theorems for Fredholm linear relations to establish a perturbation theory for Atkinson type relations.

The paper is organised as follows:

Section 1 recalls the general notation and some basic definitions from the theory of linear relations in normed spaces.

In Section 2 we describe some perturbation results for semiFredholm relations as well as some characterisation theorems for Atkinson relations which will be used extensively in Section 3.

Section 3 contains the main results. We show that with additional hypothesis, the class of $\alpha$-Atkinson relations (respectively, the class of $\beta$-Atkinson relations) is stable

Received 30th November, 2006

Copyright Clearance Centre, Inc. Serial-fee code: 0004-9727/07 \$A2.00+0.00. 
under small norm and strictly singular (respectively, small norm and strictly cosingular) perturbations.

Notations. We adhered to the notation and terminology of the book [4]: $X$ and $Y$ are infinite dimensional normed spaces over $\mathbf{K}=\mathbb{R}$ or $\mathbb{C}, B_{X}$ the closed unit ball of $X$, $X^{\prime}$ the dual space of $X$. A linear relation or multivalued linear operator $T: X \rightarrow Y$ is a mapping from a subspace $D(T) \subset X$, called the domain of $T$, into the collection of nonempty subsets of $Y$ such that $T\left(\alpha x_{1}+\beta x_{2}\right)=\alpha T\left(x_{1}\right)+\beta T\left(x_{2}\right)$ for all nonzero scalars $\alpha, \beta$ and $x_{1}, x_{2} \in D(T)$. The class of such linear relations is denoted by $L R(X, Y)$. If $T$ maps the points of its domain to singletons, then $T$ is said to be single valued or simply an operator.

Let $T \in L R(X, Y)$. The graph $G(T)$ of $T$ is defined by $G(T):=\{(x, y) \in X$ $\times Y: x \in D(T), y \in T x\}$. Let $M$ be a subspace of $D(T)$. Then $M^{\perp}:=\left\{x^{\prime} \in X^{\prime}\right.$ : $\left.x^{\prime}(m)=0, \forall m \in M\right\}$ and the restriction of $T$ to $M$, denoted by $\left.T\right|_{M}$ is defined by $G\left(\left.T\right|_{M}\right):=\{(m, y) \in X \times Y: m \in M, y \in T m\}$. For any subspace $M$ of $X$ such that $M \cap D(T) \neq \emptyset$ we write $\left.T\right|_{M \cap D(T)}=\left.T\right|_{M}$. The inverse of $T$ is the linear relation $T^{-1}$ defined by $G\left(T^{-1}\right):=\{(y, x):(x, y) \in G(T)\}$. If $T^{-1}$ is single valued, then $T$ is called injective, that is, $T$ is injective if and only if its null space $N(T):=T^{-1}(0)=\{0\}$. The subspace $T(D(T))$ denoted by $R(T)$ is called the range of $T$ and the completion $\tilde{T}$ of $T$ is defined by $G(\widetilde{T}):=\widetilde{G(T)} \subset \tilde{X} \times \tilde{Y}$ where $\tilde{X}$ denotes the completion of $X$. The conjugate or adjoint $T^{\prime}$ of $T$ is defined by $G\left(T^{\prime}\right):=G\left(-T^{-1}\right)^{\perp} \subset Y^{\prime} \times X^{\prime}$. We also define $\alpha(T):=\operatorname{dim} N(T) ; \beta(T):=\operatorname{dim} Y / R(T)$ and the index of $T$, denoted by $k(T)$, is the quantity $\alpha(T)-\beta(T)$ provided $\alpha(T)$ and $\beta(T)$ are not both infinite.

For a given closed subspace $M$ of $X$ let $Q_{M}^{X}$ or simply $Q_{M}$ denote the natural quotient map from $X$ onto $X / M$ and $J_{X}$ is the canonical injection from $X$ into its completion. We shall denote $Q_{\overline{T(0)}}$ by $Q_{T}$. Clearly $Q_{T} T$ is single valued. For $x \in D(T),\|T x\|:=\left\|Q_{T} T x\right\|$ and the norm of $T$ is defined by $\|T\|:=\left\|Q_{T} T\right\|$.

A linear relation $T \in L R(X, Y)$ is said to be closed if its graph is a closed subspace, continuous if for each open set $V$ in $R(T), T^{-1} V$ is an open set in $D(T)$ equivalently $\|T\|<\infty$, open if $T^{-1}$ is continuous equivalently $\gamma(T)>0$ where $\gamma(T)$ is the minimum modulus of $T$ defined by $\gamma(T):=\sup \{\lambda \geqslant 0: \lambda \operatorname{dist}(x, N(T)) \leqslant\|T x\|, x \in D(T)\}$, precompact if $Q_{T} T B_{D(T)}$ is totally bounded, strictly singular if there is no infinite dimensional subspace $M$ of $D(T)$ for which $\left.T\right|_{M}$ is injective and open, strictly cosingular if $\Delta^{\prime}(T)=0$ where $\Delta^{\prime}(T):=\sup \left\{\Gamma^{\prime}\left(Q_{M} T\right): M \in \mathcal{E}(Y)\right\}$ (here $\Gamma^{\prime}(T):=\inf \left\{\left\|Q_{M} J_{Y} T\right\|:\right.$ $M \in \mathcal{E}(\tilde{Y})\}$ and $\mathcal{E}(X)$ denotes the class of all closed infinite codimensional subspaces of $X), \mathcal{F}_{+}$if there exists a finite codimensional subspace $E$ of $X$ such that $\left.T\right|_{E \cap D(T)}$ is injective and open, $\phi_{+}$if $R(T)$ is closed and $\operatorname{dim} N(T)<\infty, \mathcal{F}_{-}$if $T^{\prime}$ is $\mathcal{F}_{+}$and $\phi_{-}$if $R(T)$ is closed and $\operatorname{dim} Y / R(T)<\infty$.

The class of strictly singular, $\mathcal{F}_{+}, \phi_{+}$, strictly cosingular, $\mathcal{F}_{-}$and $\phi_{-}$linear relations in $L R(X, Y)$ will be denoted by $\mathcal{S S}(X, Y), \mathcal{F}_{+}(X, Y), \phi_{+}(X, Y), \mathcal{S C}(X, Y), \mathcal{F}_{-}(X, Y)$ 
and $\phi_{-}(X, Y)$ respectively.

Following [3, 2.3], we say that a linear relation $T$ is an $\alpha$-Atkinson type relation, denoted $T \in \mathcal{A}_{\alpha}(X, Y)$, if $T \in \mathcal{F}_{+}$and $\overline{R(T)}$ is complemented in $Y . T$ is said to be a $\beta$ Atkinson type relation, denoted $T \in \mathcal{A}_{\beta}(X, Y)$, if $T \in \mathcal{F}_{-}$and $N(T)$ is complemented in $D(T)$. The class of Atkinson linear relations is defined to be $\mathcal{A}(X, Y):=\mathcal{A}_{\alpha}(X, Y)$ $\cap \mathcal{A}_{\beta}(X, Y)$.

$B(X, Y)$ denotes the class of continuous operators from $X$ into $Y$.

A single valued $S$ is said to be a linear selection of a linear relation $T$ if $T=S+T-T$ with $D(S)=D(T)$.

Finally, a closed subspace $M$ of $X$ is said to be complemented in $X$ if there exists a continuous projection $P$ defined in $X$ with $R(P)=M$. In that case $N(P)$ is said to be a complement of $M$ and we write $X=M \oplus N$ to indicate that $X=M+N$ and $M \cap N=\{0\}$.

An underlying motivation for the introduction of linear relations into operator theory by von Neumann [12] was to aid the investigation of differential equations governed by non-densely defined operators. The conjugate of such operators are linear relations. One main reason why linear relations are more convenient than operators is that one can define the inverse, the closure and the completion for a linear relation. Interesting works on multivalued linear operators include the treatise on partial differential relations by Gromov [8], the application of multivalued methods to solution of differential equations by Favini and Yagi [5], the development of fixed point theory for linear relations to the existence of mild solutions of quasi-linear differential inclusions of evolution and also to many problems of fuzzy theory (see, for example, $[1,7,11,13,15]$ ) and several papers on linear relations type semiFredholm and other classes related to them (see, for example, $[2,4]$, among others).

Throughout this paper $T$ will be an element of $L R(X, Y)$ except where stated otherwise.

\section{PRELIMINARY RESULTS}

We begin by giving some auxiliary results that we shall need to obtain the main Theorems of Section 3.

LEMma 1. Let $T \in L R(X, Y)$ be closed and let $S \in L R(X, Y)$ be continuous such that $\overline{D(T)} \subset D(S)$ and $S(0) \subset T(0)$. Then $T+S$ is closed.

Proof: Assume first that $T$ and $S$ are single valued. Let $\left(x_{n}\right)$ be a sequence in $D(T+S)=D(T) \cap D(S)=D(T)$ such that $x_{n} \rightarrow x$ and $(T+S) x_{n} \rightarrow y$ for some $x \in X$ and $y \in Y$. Then $x \in \overline{D(T)} \subset D(S)$ and $S x_{n} \rightarrow S x$ since $S$ is continuous. Thus $T x_{n} \rightarrow S x-y$ and as $T$ is closed, we have that $x \in D(T)$ and $T x=S x-y$, that is, $T+S$ is closed. For the general case, we observe that $Q_{T} T$ is a closed operator and $T(0)$ is 
closed $\left(\left[4\right.\right.$, II. 5.3]), $(T+S)(0)=T(0)$ because $S(0) \subset T(0)$ and $Q_{T} S=Q_{T(0) / \overline{S(0)}} Q_{S} S$ by [4, IV. 5.2]. Hence, from what has been proved for the single valued case, $Q_{T+S}(T+S)$ $=Q_{T} T+Q_{T} S$ is closed and since $(T+S)(0)$ is closed, applying again [4, II. 5.3] we obtain that $T+S$ is closed, as desired.

In $[4$, Chapter V], Cross proved the following properties about semiFredholm linear relations in normed spaces.

Proposition 2.

(a) The following equivalences hold:

$$
\begin{aligned}
& T \in \mathcal{F}_{+} \Leftrightarrow \tilde{T} \in \phi_{+} \Leftrightarrow T^{\prime} \in \phi_{-} \\
& T \in \mathcal{F}_{-} \Leftrightarrow \tilde{T} \in \phi_{-} \Leftrightarrow T^{\prime} \in \phi_{+} .
\end{aligned}
$$

(b) (i) If $T$ is closed and $R\left(T^{\prime}\right)$ is closed, then $k(\widetilde{T})=-k\left(T^{\prime}\right)$.

(ii) If $T$ is open, then $k(\widetilde{T})=-k\left(T^{\prime}\right)$.

(iii) If $T \in \phi_{+} \cup \phi_{-}$, then $k(\widetilde{T})=-k\left(\widetilde{T}^{\prime}\right)=-k\left(T^{\prime}\right)$.

Proposition 3. Let $T \in \mathcal{F}_{+}(X, Y)$ and suppose that $S \in L R(X, Y)$ satisfies $S(0) \subset \overline{T(0)}$. Then

(i) If $T$ is open and $\|S\|<\gamma(T)$, then $T+S$ is $\mathcal{F}_{+}$.

(ii) If $S$ is strictly singular, then $T+S$ is $\mathcal{F}_{+}$.

Proof: (i) We may clearly assume that $\operatorname{dim} D(T+S)=\infty$, so that $\operatorname{dim} D(T)=\infty$. Then by using properties of the operational quantities (not discussed here), the result follows from [4, V. 3.2 and V. 5.1].

Proposition 4. ([4, V. 5.12 and V. 5.20]) Let $T \in \mathcal{F}_{-}(X, Y)$, and suppose that $S \in L R(X, Y)$ with $D(T) \subset D(S)$. We have:

(i) If $S$ is precompact, then $T+S$ is $\mathcal{F}_{-}$.

(ii) If $\|S\|<\gamma\left(T^{\prime}\right)$, then $T+S$ is $\mathcal{F}_{-}$.

(iii) If $J_{Y} S$ is strictly cosingular and $\operatorname{dim} Q_{T} S(0)<\infty$, then $T+S$ is $\mathcal{F}_{\text {- }}$.

Proposition 5. Let $T \in \mathcal{F}_{+}(X, Y) \cup \mathcal{F}_{-}(X, Y)$ and let $S \in L R(X, Y)$ such that $\overline{D(T)} \subset D(S), S(0) \subset \overline{T(0)}$ and $\|S\|<\gamma(\widetilde{T})$. Then $k(\widetilde{T}+S)=k(\widetilde{T})$.

Proof: The proof is along the lines of the proof of the analogous result provided in $[4$, V. 15.6] for the case when $S$ is single valued.

PROPOSITION 6. Let $T \in L R(X, Y)$.

(i) Suppose $T \in \mathcal{F}_{+}(X, Y)$ and let $S \in \mathcal{S} \mathcal{S}(X, Y)$ be continuous such that $S(0) \subset \overline{T(0)}$ and $\overline{D(T)} \subset D(S)$. Then $k(\widetilde{T}+S)=k(\widetilde{T})$.

(ii) Suppose $T \in \mathcal{F}_{-}(X, Y)$, and let $S \in L R(X, Y)$ be such that $S^{\prime}$ is strictly singular. If $\left\|S^{\prime}\right\|<\infty, S(0) \subset \overline{T(0)}$ and $\overline{D(T)} \subset D(S)$, then $k(\tilde{T}+S)$ $=k(\widetilde{T})$. 
Proof: (i) By Proposition 2, $\widetilde{T} \in \phi_{+}$, and hence, we can assume that $X$ and $Y$ are complete, that $T$ is closed, and show that the stability holds for $k(T)$.

Since $T+\lambda S \in \mathcal{F}_{+}$(Proposition 3) and $T+\lambda S$ is closed (Lemma 1) is $T+\lambda S \in \phi_{+}$for all $\lambda$ (Proposition 2). Let $I:=[0,1]$ with its usual topology and let $\mathcal{Z}:=\mathbb{Z} \cup\{-\infty,+\infty\}$ with the discrete topology. Then, by substituting $T+\lambda S \in \phi_{+}$for $T$ in Proposition 5, we deduce that the map $\theta: \lambda \in I \rightarrow \theta(\lambda):=k(T+\lambda S) \in \mathcal{Z}$ is continuous and $\theta(I)$ is connected and therefore consists of only point. Thus $k(T)=\theta(0)=\theta(1)=k(T+S)$.

(ii) By Proposition 2, $\widetilde{T} \in \phi_{+}$, and hence as in (i) we can assume that $X$ and $Y$ are Banach spaces, that $T$ is closed, and show that the stability holds for $k(T)$.

Since $T+S$ is closed (Lemma 1), $(T+S)^{\prime}=T^{\prime}+S^{\prime}\left(\left[4\right.\right.$, III. 1.5]) and $\left\|S^{\prime}\right\| \leqslant\|S\|$ ([4, III. 1.13]), we obtain from (i) applied to $T^{\prime} \in \phi_{+}$that

$$
k(T)=-k\left(T^{\prime}\right)=-k\left(T^{\prime}+S^{\prime}\right)=-k\left((T+S)^{\prime}\right)=k(T+S),
$$

as desired.

THEOREM 7. ([3, 24]) Let $T \in L R(X, Y)$. If $\overline{T(0)}$ is complemented in $Y$, then the following properties are equivalent:

(i) $T \in \mathcal{A}_{\alpha}(X, Y)$.

(ii) There exists $A \in B(Y, X)$ and a finite rank projection $K \in B(X)$ such that $A$ is defined densely with $N(A)$ complemented in $Y, R(A) \subset D(T)$, $R(K) \subset D(T)$ and $A T=\left.(I-K)\right|_{D(T)}$.

TheOREM 8. ([3, 25]) Let $T \in L R(X, Y)$. If $N(T)$ is closed in $D(T)$, then the following properties are equivalent:

(i) $T \in \mathcal{A}_{\beta}(X, Y)$.

(ii) There exists $B \in B(Y, X)$ and a finite rank projection $K \in B(Y)$ such that $B$ is everywhere defined, $R(B)$ is complemented in $D(T), B T$ and $K T$ are continuous operators and $(I-K)$ is a linear selection of $T B$.

We refer to an operator $A \in B(Y, X)$ satisfying Theorem 7 (ii) as a left regulariser or left generalised inverse of $T \in \mathcal{A}_{\alpha}$, and refer to an operator $B \in B(Y, X)$ satisfying Theorem 8 (ii) above as a right regulariser or right generalised inverse of $T \in \mathcal{A}_{\beta}$.

The left generalised inverse $A$ of $T \in \mathcal{A}_{\alpha}(X, Y)$ is continuous and everywhere defined when the spaces $X$ and $Y$ are complete and $T$ is closed (see, [3, 24]).

REMARK 9. ([3, 23]) In Theorem 7 above, it is suffices to consider the proof applying to the case when $I-K$ is $\mathcal{F}_{+}$in the place of having $K$ precompact. Similarly, in the proof of Theorem 8 it is suffices to consider the case $(I-K) \in \mathcal{F}_{+} \cap \mathcal{F}_{\text {- instead of } K}$ precompact. 


\section{Perturbation of atkinson linear relations}

In this section we investigate the stability of Atkinson linear relations under small norm, strictly singular and strictly cosingular perturbations, as well as the behaviour of the index under perturbation.

TheOREM 10. Let $T \in L R(X, Y)$ and suppose $S \in L R(X, Y)$ satisfies $\overline{D(T)}$ $\subset D(S)$ and $S(0) \subset \overline{T(0)}$.

(i) If $T \in \mathcal{A}_{\alpha}(X, Y)$ and $\overline{T(0)}$ is complemented in $Y$, then there exists $\varepsilon>0$ such that $\|S\|<\varepsilon$ implies $T+S \in \mathcal{A}_{\alpha}(X, Y)$.

(ii) Let $T \in \mathcal{A}_{\beta}(X, Y)$ such that $N(T)$ is closed in $D(T),(T+S)(0)$ and $N(T+S)$ are closed in $R(T+S)$ and $D(T+S)$ respectively. Then there exists $\varepsilon>0$ such that $\|S\|<\varepsilon$ implies $T+S \in \mathcal{A}_{\beta}(X, Y)$.

(iii) If (i) or (ii) holds and $\|S\|<\gamma(\tilde{T})$, then $k(\tilde{T}+S)=k(\tilde{T})$.

Proof: (i) By Theorem 7, $A T=\left.\left(I-K_{A T}\right)\right|_{D(T)}$ where $K_{A T}$ is the finite rank projection operator associated with $T \in \mathcal{A}_{\alpha}$ and the given left regulariser $A$. Therefore $0<\gamma\left(I-K_{A T}\right) \leqslant \gamma\left(\left.\left(I-K_{A T}\right)\right|_{N\left(I-K_{A T}\right)+D(T)}\right)\left([4\right.$, II. 6.1] $)=\gamma\left(\left(I-\left.K_{A T}\right|_{D(T)}\right)\right.$ (as $N\left(I-K_{A T}\right)=R\left(K_{A T}\right) \subset D(T)$ by Theorem 7) and thus $\gamma(A T)>0$. Let $\varepsilon:=(\gamma(A T) / \|$ $A \|)>0$. By Theorem 7,

$$
A(T+S)=A T+A S=\left.\left(I-K_{A T}+A S\right)\right|_{D(T)}
$$

Since $\|A S\| \leqslant\|A\|\|S\|\left(\left[4\right.\right.$, II. 3.13]) $<\gamma(A T)$, on perturbing $I-K_{A T}$ by $A S$, it follows from Proposition 3 that $A(T+S) \in \mathcal{F}_{+}$. Moreover, from Theorem 7 it is clear that $R(A) \subset D(T)=D(T+S), R\left(K_{A T}-A S\right) \subset D(T+S)$ and $\overline{(T+S)(0)}=\overline{T(0)}$ (as $S(0) \subset \overline{T(0)}$ ) is complemented in $Y$. Hence, applying Theorem 7 to $T+S$ with $A$ serving as a left generalised inverse, and substituting $K_{A T}-A S$ for the operator $K$ in the same Theorem, it follows from Remark 9 that $T+S \in \mathcal{A}_{\alpha}(X, Y)$.

(ii) We first show that the assertion holds for the case when $T$ and $S$ are single valued. By Theorem $8, T B=I-K_{T B}$ where $K_{T B}$ is the finite rank projection operator associated with $T \in \mathcal{A}_{\beta}$ and the given right regulariser $B$. Thus $T B$ is open. Let $\varepsilon:=(\gamma(T B) /\|B\|)>0$. Then,

$$
(T+S) B=T B+S B=I-K_{T B}+S B
$$

On perturbing $I-K_{T B} \in \mathcal{F}_{+} \cap \mathcal{F}_{-}$by $S B$, it follows from Propositions 3 and 4 that $(T+S) B \in \mathcal{F}_{+} \cap \mathcal{F}_{-}$. By Theorem $8, R(B)$ is complemented in $D(T+S)=D(T)$, $B(T+S)=B T+B S$ and $K_{T B}(T+S)=K_{T B} T+K_{T B} S$ are continuous operators. Thus, applying Theorem 8 to $T+S$ with $B$ serving as a right regulariser and substituting $K_{T B}-S B$ for the operator $K$ in the same Theorem, it follows from Remark 9 that $T+S \in \mathcal{A}_{\beta}(X, Y)$. 
Passing to the general case, let $B_{Q}:=B Q_{T}^{-1} \in L R(Y / \overline{T(0)}, X)$. Thus, for $[y] \in Y / \overline{T(0)}$, we have $B_{Q}[y]=B(y+\overline{T(0)}) \subset B y+\overline{B T(0)}=B y$ (since $B T$ is single valued). Hence, $B_{Q}$ is single valued. Define $K_{Q} \in L R(Y / \overline{T(0)})$ by $K_{Q}[y]:=[K y]$. It is easy to verify that $Q_{T} T B_{Q}=I_{Y / \overline{T(0)}}-K_{Q}$ and $Q_{T} T, B_{Q}$ and $K_{Q}$ satisfy the hypothesis of Theorem 8 (ii). Furthermore, $Q_{T+S}(T+S) B_{Q}=Q_{T}(T+S) B_{Q}\left(\right.$ as $\left.S(0) \subset \overline{T(0)} \Rightarrow Q_{T}=Q_{T+S}\right)$ $=Q_{T} T B_{Q}+Q_{T} S B_{Q}$. In consequence

$$
Q_{T+S}(T+S) B_{Q}=I_{Y / \overline{T(0)}}-K_{Q}+Q_{T} S B_{Q}
$$

Now, assume that $\|S\|<\gamma\left(Q_{T} T B_{Q}\right) /\left\|B_{Q}\right\|$. Then, from what has been proved for the single valued case, it follows that $Q_{T+S}(T+S) \in \mathcal{F}_{-}$and $N\left(Q_{T+S}(T+S)\right)$ is

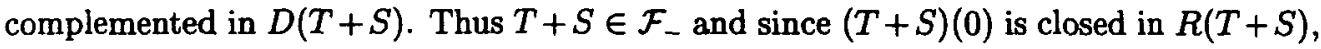
we have that $N\left(Q_{T+S}(T+S)\right)=N(T+S)$ by [4, II. 3.4]. The result follows.

(iii) This follows immediately from Proposition 5.

TheOREM 11. Let $T \in \mathcal{A}_{\alpha}(X, Y)$ with $\overline{T(0)}$ complemented in $Y$, and suppose $S \in \mathcal{S S}(X, Y)$ such that $\overline{D(T)} \subset D(S)$ and $S(0) \subset \overline{T(0)}$. Then

(i) $T+S \in \mathcal{A}_{\alpha}(X, Y)$.

(ii) If $S$ is continuous, then $k(\widetilde{T}+S)=k(\widetilde{T})$.

Proof: By Theorem 7, $A T=\left.\left(I-K_{A T}\right)\right|_{D(T)}$ where $K_{A T}$ is the finite rank projection associated with $T \in \mathcal{A}_{\alpha}$ and the given left generalised inverse $A$. Since $A S$ is strictly singular ([4, V. 2.10]), we have

$$
A(T+S)=\left.\left(I-K_{A T}+A S\right)\right|_{D(T)} .
$$

On perturbing $I-K_{A T}$ by $A S$, it follows from Proposition 3, that $A(T+S) \in \mathcal{F}_{+}$. As in Theorem 10 (i) we have $R(A) \subset D(T+S)$ and $R\left(K_{A T}-A S\right) \subset D(T+S)$. Furthermore, $\overline{(T+S)(0)}$ is complemented in $Y$ since $\overline{(T+S)(0)}=\overline{T(0)}($ as $S(0) \subset \overline{T(0)})$. Now, applying Theorem 7 to $T+S$ with $A$ serving as a left regulariser, and substituting $K_{A T}-A S$ for the operator $K$ in the same Theorem, we conclude from Remark 9 that $T+S \in \mathcal{A}_{\boldsymbol{\alpha}}$.

(ii) This assertion follows immediately from Proposition 6 (i).

In Theorem 11, the proof of stability of the index was restricted to perturbation by continuous strictly singular relations. Using the graph norm, we show that this result can be extended to strictly singular linear relations which are relatively bounded.

Definition 12: ([4, VII. 2.1]) A linear relation $S \in L R(X, Y)$ is said to be $T$ bounded if $D(T) \subset D(S)$ and there exists $a, b>0$ for which the inequality

$$
\|S x\| \leqslant a\|x\|+b\|T x\|
$$

holds for all $x \in D(T) . S$ is called $T$-precompact (respectively, $T$-strictly singular) if $S$ is precompact (respectively, strictly singular) with respect to the graph norm defined in 
$D(T)$ by

$$
\|x\|_{T}:=\|x\|+\|T x\|, \quad x \in D(T) .
$$

THEOREM 13. Let $X$ and $Y$ be complete, and let $T \in \mathcal{A}_{\alpha}(X, Y)$ be closed with $T(0)$ complemented in $Y$. If $S$ satisfies $\overline{D(T)} \subset D(S), S(0) \subset T(0)$, and $S$ is $T$-bounded and $T$-strictly singular, then $T+S \in \mathcal{A}_{\alpha}(X, Y)$ and $k(T+S)=k(T)$.

Proof: Since $X$ and $Y$ are complete and $T$ is closed, the space $X_{T}:=\left(D(T), \|\right.$. $\left.\|_{T}\right)$ is also a Banach space. Let $T_{T}, S_{T} \in L R\left(X_{T}, Y\right)$ be defined by $T_{T} x:=T x$ and $S_{T} x:=S x$ for $x \in X_{T}$. Clearly $S_{T}$ is continuous with $\left\|S_{T}\right\| \leqslant 1$. Thus $T_{T}+S_{T}$ is closed by Lemma 1 . If $U \in L R\left(D(T), X_{T}\right)$ denotes the identity map from $D(T)$ onto $X_{T}$, then $U$ is continuous and $T+S=\left(T_{T}+S_{T}\right) U$ is closed by [4, II. 5.18]. By Theorem 7, $A T=I-K$ where $K$ denotes the finite rank projection associated to $T$ and the given left regulariser $A$ which is continuous and everywhere defined.

Let $A_{T} \in L R\left(Y, X_{T}\right), K_{T} \in L R\left(X_{T}\right)$ be defined by $K_{T} x:=K x$ for $x \in X_{T}$ and $A_{T} y:=A y$ for $y \in Y$. We shall prove the following properties:

$T A$ is continuous

Indeed, $T A$ is closed by [4, II. 5.18] with $D(T A)=Y$ (since $R(A) \subset D(T)$ ) and thus it follows from the Closed Graph Theorem for linear relations[4, II. 5.4], that $T A$ is continuous.

$$
A_{T} \text { is continuous }
$$

By (1) it follows that

$$
\begin{gathered}
\left\|A_{T} y\right\|_{T}=a\|A y\|+b\|T A y\| \leqslant(a\|A\|+b\|T A\|)\|y\|, \quad y \in Y . \\
T_{T} \in \mathcal{A}_{\alpha}\left(X_{T}, Y\right)
\end{gathered}
$$

This property is deduced trivially from Theorem 7, upon noting that $N\left(T_{T}\right)=N(T)$, $R\left(T_{T}\right)=R(T)$ and $A_{T}$ and $K_{T}$ satisfy the conditions of the Theorem 7 (ii).

In this situation, by virtue of (3), $T_{T}$ and $S_{T}$ verify the hypothesis of Theorem 11 and so the desired result follows immediately since $R(T+S)=R\left(T_{T}+S_{T}\right)$ and $N(T+S)=N\left(T_{T}+S_{T}\right)$.

THEOREM 14. Let $T, S \in L R(X, Y)$ such that $N(T)$ is closed in $D(T), \overline{D(T)}$ $\subset D(S), S(0) \subset \overline{T(0)},(T+S)(0)$ and $N(T+S)$ are closed in $R(T+S)$ and $D(T+S)$ respectively. If $T \in \mathcal{A}_{\beta}(X, Y), S \in \mathcal{S S}(X, Y), J_{Y} S \in \mathcal{S C}(X, \tilde{Y})$ and $\operatorname{dim} Q_{T} S(0)<\infty$, then

(i) $T+S \in \mathcal{A}_{\beta}(X, Y)$.

(ii) If $S^{\prime}$ is continuous and strictly singular, then $k(\widetilde{T}+S)=k(\widetilde{T})$. 
Proof: (i) Let us consider two cases for $T$ and $S$ :

CASE I. $T$ and $S$ are single valued. Let $B$ denote a continuous right regulariser of $T$. Then $S B$ is strictly singular ([4, V. 2. 12]), $J_{Y} S B$ is strictly cosingular $([4$, IV. 5.8]) and we obtain by Theorem 8 that

$$
(T+S) B=I-K_{T B}+S B
$$

where $K_{T B}$ is the finite rank projection operator associated with $T \in \mathcal{A}_{\beta}$. Hence, on perturbing $I-K_{T B} \in \mathcal{F}_{+} \cap \mathcal{F}_{-}$with $S B$ it follows from Propositions 3 and 4 that $(T+S) \in \mathcal{F}_{+} \cap \mathcal{F}_{-}$. Furthermore, as $S$ is continuous we deduce from Theorem 8 that $R(B)$ is complemented in $D(T+S), B(T+S)=B T+B S$ and $K_{T B}(T+S)=K_{T B} T+K_{T B} S$ are continuous operators. In this situation, applying Theorem 8 to $T+S$ with $B$ serving as a right generalised inverse, and substituting $K_{T B}-S B$ for the operator $K$ in the same Theorem, it follows from Remark 9 that $T+S$ is a $\beta$-Atkinson operator.

CASE II. $T$ and $S$ are linear relations. We adopt the notation of the proof of Theorem 10 (ii) and arguing exactly as in the same proof we obtain

$$
Q_{T+S}(T+S) B_{Q}=I-K_{Y / \overline{T(0)}}+Q_{T} S B_{Q}
$$

Moreover, we shall now show that

$Q_{T} S B_{Q}$ is a strictly singular operator.

This property follows trivially from [4, V. 2.11 and V. 2. 12].

$J_{Y / \overline{T(0)}} Q_{T} S$ is a strictly cosingular operator.

Indeed, we may clearly suppose that $\operatorname{dim} Y / \overline{T(0)}=\infty$. Let $\widehat{T(0)}$ denote the closure of $\overline{T(0)}$ considered as a subspace of $\tilde{Y}$.

From the canonical equality $\tilde{Y} / \widehat{T(0)}=\widetilde{Y / \overline{T(0)}}$, the operator $J_{Y / \overline{T(0)}} Q_{T}$ is naturally identified with $Q_{\widehat{T(0)}} J_{Y}$. In consequence, $J_{Y / \overline{T(0)}} Q_{T} S=Q_{\widehat{T(0)}} J_{Y} S$ and then an application of $[4$, IV. 5.4] yields immediately the desired property.

In this situation, substituting $Q_{T} S B_{Q}$ for $S B, B_{Q}$ for $B$ and $Q_{T+S}(T+S)$ for $T+S$ the result follows from the Case I, upon noting that $N\left(Q_{T+S}(T+S)\right)=N(T+S)$ since $(T+S)(0)$ is closed in $R(T+S)$.

(ii) This assertion is clear from Proposition 6 (ii).

\section{REFERENCES}

[1] R.P. Agarwal, M. Mehan and D. O'Regan, Fixed point theory and applications (Cambridge University Press, Cambridge, London, 2001). 
[2] T. Álvarez, 'On almost semi-Fredholm linear relations in normed spaces', Glasgow Math. J. 47 (2005), 187-193.

[3] T. Álvarez, R.W. Cross and D. Wilcox, 'Multivalued Fredholm type operators with abstract generalised inverses', J. Math. Anal. Appl. 261 (2001), 403-417.

[4] R.W. Cross, Multivalued linear operators (Marcel Dekker, New York, 1998).

[5] A. Favini and A. Yagi, 'Multivalued linear operators and degenerate evolution equations', Ann. Mat. Pura. Appl. (4) 163 (1993), 353-384.

[6] M. González, 'On Atkinson operators in locally convex spaces', Math. Z. 190 (1985), 505-517.

[7] L. Gorniewicz, Topological fixed point theory of multivalued mappings (Kluwer, Dordrecht, 1999).

[8] M. Gromov, Partial differential relations (Springer-Verlag, Berlin, 1966).

[9] T. Kato, 'Perturbation theory for nullity, deficiency and other quantities of linear operators', J. Anal. Math. 6 (1958), 261-322.

[10] V. Müller-Hörrig, 'Zur theorie der semi-Fredolm-operatoren mit stetig projizierten Kern und Bild', Math. Nach. 99 (1980), 185-197.

[11] M. Muresan, 'On a boundary value problem for quasi-linear differential inclusions of evolution', Collect. Math. 45 (1994), 165-175.

[12] J. von Neumann, Functorial operators Vol. 2, The Geometry of Orthogonal Spaces Annals of Math. Studies 22 (Princeton University Press, Princeton N. J., 1950).

[13] H.A. Román-Flores, A. Flores-Franulic, M.A. Rojas-Medar and R.C. Bassanezi, 'Stability of the fixed points set of fuzzy contractions', Appl. Math. Lett. 11 (1998), 33-37.

[14] J.I. Vladimirskii, 'Strictly cosingular operators', Soviet Math. Dokl. 8 (1967), 739-740.

[15] Z. Yuanguo and R. Ling, 'Differential inclusions for fuzzy maps', Fuzzy sets and Systems 112 (2000).

Department of Mathematics

University of Oviedo

33007, Oviedo

Asturias

Spain

e-mail: seco@uniovi.es
Department of Mathematics and Applied Mathematics

University of Cape Twon

Rondebosch, 7700

South Africa

e-mail: diane@maths.uct.ac.za 